\title{
Nicht pegyliertes liposomales Doxorubicin doch nicht weniger kardiotoxisch?
}

Die längere Anwendung von Anthrazyklinen ist durch die kumulative, dosisabhängige Kardiotoxizität limitiert, vor allem bei Patienten mit metastasierten Tumoren, die bereits mit Anthrazyklinen vorbehandelt wurden. In der Myotax-Studie ging man der Frage nach, ob nicht pegyliertes liposomales Doxorubicin (NPLD) weniger riskant fürs Herz ist.

$\mathrm{N}$ icht pegyliertes liposomales Doxorubicin (NPLD) hat eine ebenso gute Anti-Tumor-Aktivität wie konventionelles Doxorubicin, aber eine signifikant niedrigere Kardiotoxizität, wenn es als Einzelsubstanz oder in Kombination mit Cyclophosphamid verabreicht wird. In der Phase-II-Studie wurden die Wirksamkeit und Sicherheit von NPLD in Kombination mit Docetaxel bei Patientinnen mit metastasiertem Brustkrebs untersucht, die bereits eine adjuvante Therapie mit Anthrazyklinen erhalten hatten. 34 Patientinnen mit metastasiertem Brustkrebs erhielten NPLD $\left(60 \mathrm{mg} / \mathrm{m}^{2}\right)$ und Docetaxel $\left(75 \mathrm{mg} / \mathrm{m}^{2}\right)$. Dabei waren sechs 21-Tage-Zyklen vorgesehen. Die Behandlung erfolgte bis zum Fortschreiten der Erkrankung oder bis zum Auftreten von inakzeptablen Toxizitäten.

Die objektive Ansprechrate betrug $79 \%$, wobei $27 \%$ der Patientinnen eine vollständige Response zeigten. Das mediane progressionsfreie Überleben betrug 11,3 Monate und das mediane Gesamtüberleben 28,2 Monate.

$47 \%$ der Patientinnen litten unter febriler Neutropenie. Überraschend war jedoch, dass so viele kardiale Nebenwirkungen auftraten. $15 \%$ der Patientinnen hatten Symptome einer Herzinsuffizienz, die einen Therapieabbruch erforderlich machten. Die kardialen Symptome traten im Median nach 18,2 Wochen auf. Eine Patientin starb zwei Jahre später an einer kardialen Komplikation. Eine Patientin brach die Therapie wegen kardialer Nebenwirkungen vom Grad 2 ab. Die kumulative Dosis von Doxorubicin bot keine Erklärung für die hohe Kardiotoxizität. Frühere Studien hatten eine geringere Kardiotoxizität ergeben.

Fazit: Die Kombination von NPLD und Docetaxel zeigte hohe Anti-Tumor-Aktivität bei Patientinnen mit metastasiertem Brustkrebs, die bereits eine adjuvante

Warum sind Anthrazykline kardiotoxisch?

Dosisunabhängig verursachen Anthrazykline eine reversible frühe Kardiotoxizität mit ST-Streckenveränderungen und Rhythmusstörungen. Mit steigender Dosierung kann aber auch Jahre nach einer Therapie die nicht reversible ¿ Anthrazyklin-induzierte Kardiomyopathie auftreten. Diese entsteht, indem Anthrazykline eine Lipidperoxidation der Mitochondrienmembran von Herzmuskelzellen auslösen, die zur Myokardfibrose führen kann.

Therapie mit Anthrazyklinen erhalten hatten. Allerdings trat eine unerwartet hohe Rate kardialer Nebenwirkungen auf. Judith Neumaier

Curtit E et al. Myotax: A phase II trial of docetaxel plus non-pegylated liposomal doxorubicin as first-line therapy of metastatic breast cancer previously treated with adjuvant anthracyclines. Eur J Cancer. 2011;47: 2396-402.

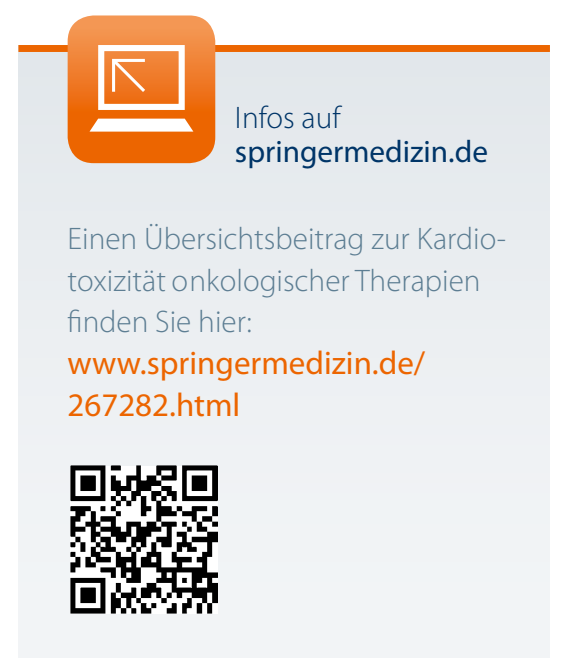

